\title{
The Incidence of Atrial Fibrillation after On-Pump Versus Off-Pump Coronary Artery Bypass Grafting
}

\author{
Gokhan Arslan, ${ }^{1}$ Gokhan Erol, ${ }^{2}$ Hakan Kartal, ${ }^{2}$ Ertan Demirdas, ${ }^{2}$ Cengiz Bolcal ${ }^{1}$ \\ ${ }^{1}$ University of Health Sciences, Gulhane Faculty of Medicine, Department of Cardiovascular Surgery, Ankara, Turkey; ${ }^{2}$ Gulhane Training \\ and Research Hospital, Ankara, Turkey
}

\section{ABSTRACT}

Background: This study aimed to investigate the incidence of postoperative atrial fibrillation (POAF) in patients undergoing off-pump versus on-pump coronary artery bypass grafting (CABG) under cardiopulmonary bypass $(\mathrm{CPB})$.

Methods: A total of 3,197 consecutive patients $(1,816$ males, 1,381 females; mean age: $60.8 \pm 9.8$ years) with preoperative sinus rhythm who underwent CABG at a cardiovascular surgery clinic between November 2009 and March 2014 retrospectively were analyzed. Of the patients, 1,680 underwent on-pump and 1,517 underwent off-pump cardiac surgery. Data, including demographic characteristics, preoperative risk factors, preoperative medications, laboratory test results, postoperative data and complications, and mortality and morbidity rates, were recorded.

Results: According to the multivariate analysis, the type of operation, number of anastomoses, right coronary artery or right coronary posterior descending artery graft, vasopressor therapy (epinephrine, norepinephrine), operation duration, age $>60$ years, hypertension, length of hospital stay $>4$ days, and obstructive sleep apnea syndrome (OSAS) were the independent predictors of POAF after CABG. Our study results suggest that on-pump $\mathrm{CABG}$ under $\mathrm{CPB}$ is correlated with POAF.

Conclusion: We recommend using off-pump CABG in select cases to minimize the risk of POAF.

\section{INTRODUCTION}

Atrial fibrillation $(\mathrm{AF})$, which is the most common cardiac arrhythmia, is a major public health problem leading to increased health-related costs due to the aging population. The prevalence of $\mathrm{AF}$ is estimated as $2 \%$ in the general population [Zoni-Berisso 2014]. Postoperative AF (POAF) is the most frequent cardiac complication, following coronary artery bypass grafting (CABG), accounting for $15 \%$ to $50 \%$ of cases [Maesen 2012].

Received April 8, 2021; accepted May 10, 2021.

Correspondence: Gokhan Arslan, Asst. Prof., University of Health Sciences, Gulhane Faculty of Medicine, Department of Cardiovascular Surgery, 06010 Etlik, Ankara, Turkey; +90-312-304-52-14; fax: +90-312-304-27-00 (e-mail:drgokhanarslan@gmail.com).
The majority of AF in the early postoperative period is temporary and can spontaneously resolve. Atrial fibrillation following CABG was found to be associated with hemodynamic instability, prolonged hospital stays, an increased risk for thromboembolic events, and early and late mortality [Maesen 2012].

Although the etiology of POAF still is not elucidated, several authors have suggested that cardiopulmonary bypass $(\mathrm{CPB})$ may be correlated with POAF. The most common culprits blamed for POAF include right atrial manipulation, cannulation, cardioplegic arrest, prolonged cross-clamping, insufficient protection of the atrial tissue from ischemia, the release of CPB-related inflammatory mediators, and oxidative stress [Sabzi 2011; Hogue 2000; Gasparova 2017].

There is no consensus on the risk factors of POAF. Therefore, we aimed to investigate the incidence of POAF in patients undergoing off-pump versus on-pump CABG under $\mathrm{CPB}$ in the present study.

\section{MATERIALS AND METHODS}

This retrospective study included 3,197 consecutive patients (1,816 males, 1,381 females) with preoperative sinus rhythm and no history of AF who underwent $\mathrm{CABG}$ at a cardiovascular surgery clinic between November 2009 and March 2014. Patients with a history of AF development despite preoperative sinus rhythm, emergency CABG, urgent CABG, additional procedures with CABG, reoperation, and open cardiac surgery except CABG were excluded from the study. The only interest of outcome was postoperative AF, thereby, other types of arrhythmias, such as supraventricular arrhythmias other than $\mathrm{AF}$ and ventricular arrhythmias, were excluded. However, atrial flutter was accepted as AF since they were in the same spectrum of arrhythmia development and the treatment protocol was the same. The written informed consent was obtained from each patient. The study protocol was approved by the Lokman Hekim University Ethics Committee (25.12.2019-2019038). The study was conducted, according to the principles of the Declaration of Helsinki.

Data, including demographic characteristics, preoperative risk factors, preoperative medications, laboratory test results, postoperative data, and complications and mortality and morbidity rates, were obtained from hospital records and hospital databases. Additionally, postoperative intubation, length of intensive care unit (ICU) and hospital stay, comorbidities such 
Table 1. Baseline demographic and clinical characteristics of patients

\begin{tabular}{lccc}
\hline Variable & $\begin{array}{c}\text { Off-Pump CABG } \\
(\mathrm{N}=1,517)\end{array}$ & $\begin{array}{c}\text { On-Pump CABG } \\
(\mathrm{N}=1,680)\end{array}$ & $P$ \\
\hline Age & $61.63 \pm 9.57$ & $60.2 \pm 10.17$ & .752 \\
Sex (male/female) & $803(53 \%) / 514$ & $\begin{array}{c}1013(60 \%) / 867 \\
(47 \%)\end{array}$ & $.041 *$ \\
Diabetes mellitus & $417(27 \%)$ & $576(34 \%)$ & .071 \\
Hypertension & $872(57 \%)$ & $1124(67 \%)$ & $.001 *$ \\
COPD & $231(15 \%)$ & $324(19 \%)$ & .425 \\
Previous myocardial & $496(33 \%)$ & $768(46 \%)$ & $.001 *$ \\
infarction & $29.2 \pm 3.8$ & $29.8 \pm 4.1$ & .361 \\
Body mass index $\left(\mathrm{kg} / \mathrm{m}^{2}\right)$ & $46.7 \pm 8.4$ & $48.3 \pm .9 .2$ & .245 \\
LVEF (mean \pm SD) & $3.7 \pm 2.4$ & $3.4 \pm 2.2$ & .123 \\
Standard EuroSCORE & & & \\
\hline
\end{tabular}

Data are given in mean \pm SD or in number. COPD, chronic obstructive pulmonary disease; LVEF, left ventricular ejection fraction; EuroSCORE, European System for Cardiac Operative Risk Evaluation

as diabetes mellitus, hypertension, and chronic obstructive pulmonary disease, risk factors, preoperative arrhythmia, and echocardiographic findings, coronary angiography findings, the number of vessels bypassed, type of grafts used, duration of aortic cross-clamp and total CPB, the total amount of cardioplegia, postoperative drainage and total amount of blood products, and postoperative medications used in patients with POAF were evaluated. All patients underwent 6-channel, 5-lead D-II electrocardiography (ECG) and invasive arterial monitoring through the radial artery for three days in the intensive care unit. Moreover, the patients were administered blood gas analysis for acid-base imbalance, electrolyte imbalance, partial oxygen, and carbon dioxide pressure hourly on the first day and every four hours on the second day and thereafter. The patients with hemodynamic stability who were transferred to the ward after the drain tubes were removed were followed through body temperature and pulse examination, arterial pressure, and oxygen saturation using a finger probe. All patients underwent standard 12-lead ECG every day, during their intensive care unit and hospital stay. The patients with postoperative arrhythmia also underwent standard 12-lead ECG during routine follow up. Postoperatively, arterial blood gas (serum $\mathrm{K}+, \mathrm{PaO} 2, \mathrm{PaCO}$, etc.) was checked during each episode of $\mathrm{AF}$ and corrected before starting further therapy. Hemodynamically stable patients were treated with intravenous amiodarone and low molecular weight heparin (LMWH). In addition, cardioversion was used if ventricular rate control was not achieved within 48 hours of initiation of therapy.

\section{Operative technique}

Off-pump CABG was performed on patients with high risk or contraindication for $\mathrm{CPB}$ and aortic cross-clamp, those with severe atheromatous plaques and dense calcifications of
Table 2. Intraoperative data of patients

\begin{tabular}{lccc}
\hline & $\begin{array}{c}\text { Off-Pump CABG } \\
\text { Variable }\end{array}$ & $\begin{array}{c}\text { On-Pump CABG } \\
(\mathrm{N}=1,517)\end{array}$ & $P$ \\
\hline $\begin{array}{l}\text { Cross-clamp time (min) } \\
\begin{array}{l}\text { Cardiopulmonary bypass } \\
\text { time (min) }\end{array}\end{array}$ & $\mathrm{NA}$ & $67.2 \pm 23.5$ & - \\
$\begin{array}{l}\text { Operation duration (min) } \\
\begin{array}{l}\text { Total amount of } \\
\text { cardioplegia (mL) }\end{array}\end{array}$ & $138.25 \pm 29.12$ & $227.13 \pm 49.17$ & .001 * \\
$\begin{array}{l}\text { Number of anastomosis } \\
\quad \text { N2 }\end{array}$ & $1296(85 \%)$ & $724(43 \%)$ & $.001 *$ \\
$\quad 221(15 \%)$ & $956(57 \%)$ & $.001 *$ \\
$\begin{array}{l}\text { Diffuse coronary artery } \\
\text { disease }\end{array}$ & $227(15 \%)$ & $1120(67 \%)$ & $.001 *$ \\
$\begin{array}{l}\text { Left internal mammary } \\
\text { artery use }\end{array}$ & $1515(99 \%)$ & $1660(99 \%)$ & .981 \\
$\begin{array}{l}\text { Right coronary artery or } \\
\text { right coronary posterior } \\
\text { descending artery graft }\end{array}$ & $287(19 \%)$ & $1163(69 \%)$ & $.001 *$ \\
\hline
\end{tabular}

Data are given in mean \pm SD or in number. NA, not applicable

the ascending aorta, aortic disease with a high-risk for embolization, rupture, or dissection, renal impairment or chronic renal failure, a history of transient ischemic attack or cerebrovascular disease, respiratory problems, systemic disease or comorbidities complicating surgery and elderly patients. On-pump CABG was performed on patients with poor target vessel quality, intramyocardial, diffuse coronary artery disease, hemodynamic instability, severe left ventricular dysfunction, recent myocardial infarction, and those who were scheduled for endarterectomy. All operations were performed through a median sternotomy. The technique used in the offpump CABG was previously described in detail by Yanagawa and Puskas [Yanagawa 2016].

On-pump CABG was performed using aorta caval cannulation. Two patients, who underwent off-pump CABG and required urgent $\mathrm{CPB}$ during surgery due to hemodynamic instability, were switched to on-pump CABG. Following cardiac arrest using antegrade and retrograde cold crystalloid cardioplegia and topical hypothermia, intermittent retrograde cold blood cardioplegia was administered to all patients. All operations were completed under moderate hypothermia $\left(32^{\circ} \mathrm{C}\right.$ to $\left.35^{\circ} \mathrm{C}\right)$. The left internal mammary artery (LIMA) grafts were used for the left anterior descending artery in 3,175 patients, while the great saphenous vein was harvested for other operations. The patients were provided warm blood cardioplegia before cross-clamp removal.

\section{Data analysis}

Data were analyzed using SPSS version 13.0 software program (SPSS Inc., Chicago, IL, USA). Descriptive statistics 
Table 3. Postoperative data of patients

\begin{tabular}{|c|c|c|c|}
\hline Variable & $\begin{array}{l}\text { Off-Pump CABG } \\
\qquad(N=1,517)\end{array}$ & $\begin{array}{l}\text { On-Pump CABG } \\
(N=1,680)\end{array}$ & $P$ \\
\hline $\begin{array}{l}\text { Vasopressor therapy (epi- } \\
\text { nephrine, norepinephrine) }\end{array}$ & $73(5 \%)$ & $227(14 \%)$ & $.002 *$ \\
\hline $\begin{array}{l}\text { Positive inotropic support } \\
\text { (dopamine) }\end{array}$ & $176(12 \%)$ & $462(28 \%)$ & $.001 *$ \\
\hline Intra-aortic balloon pump & $12(1 \%)$ & $65(4 \%)$ & $.021 *$ \\
\hline Chest tube drainage $(\mathrm{mL})$ & $487.6 \pm 182.3$ & $692.5 \pm 256.4$ & $.001 *$ \\
\hline $\begin{array}{l}\text { Total amount of blood } \\
\text { products }(\mathrm{mL})\end{array}$ & $523.3 \pm 164.7$ & $738.5 \pm 274.8$ & $.001 *$ \\
\hline Intubation duration (hour) & $6.3 \pm 1.7$ & $10.4 \pm 3.2$ & $.001 *$ \\
\hline Length of ICU stay (day) & $2.2 \pm 1.1$ & $3.4 \pm 1.5$ & $.001 *$ \\
\hline Length of hospital stay (day) & $5.2 \pm 2.4$ & $8.1 \pm 3.7$ & $.002 *$ \\
\hline $\begin{array}{l}\text { Time to POAF development } \\
\text { (hour) }\end{array}$ & $34.9 \pm 12.4$ & $36.4 \pm 13.9$ & .498 \\
\hline Re-exploration & $12(1 \%)$ & $76(5 \%)$ & $.042 *$ \\
\hline Mortality & $2(0.1 \%)$ & $17(1 \%)$ & .082 \\
\hline
\end{tabular}

Data are given in mean \pm SD or in number. NS, not significant; POAF, postoperative atrial fibrillation; ICU, intensive care unit

were expressed in mean \pm standard deviation $(\mathrm{SD})$, median (min-max), or number and frequency for continuous variables. Categorical variables were compared between the groups using the Chi-square test. The Fisher exact test was used for the expected contingency table cells with a frequency less than 5. The Mann-Whitney $U$ test and independent samples t-test were used to analyze significant differences between the groups for continuous variables. The relationship between the presence of AF and pre-, intra-, and postoperative variables was analyzed through the univariate analysis. Variables that were found to be significant in the univariate analysis with a $P$-value of $<.25$ were included in the multivariate logistic regression analysis and independent predictors of AF were determined. A $P$-value of $<.05$ with a $95 \%$ confidence interval (CI) was considered significant.

\section{RESULTS}

Of the patients, 1,680 underwent on-pump and 1,517 underwent off-pump CABG. The baseline demographic and clinical characteristics of the patients are shown in Table 1.

Of all 3,197 patients, 635 (19.86\%) developed POAF, including 163 patients $(10.74 \%)$ in the off-pump group and 472 patients $(29.09 \%)$ in the on-pump group. There was a significant difference in sex $(P=.041)$, hypertension $(P=$ $.001)$, previous myocardial infarction $(P=.001)$, operation duration $(P=.001)$, number of anastomosis $(P=.001)$, diffuse coronary artery disease $(P=.001)$, right coronary artery graft $(P=.001)$, vasopressor therapy (epinephrine, norepinephrine)

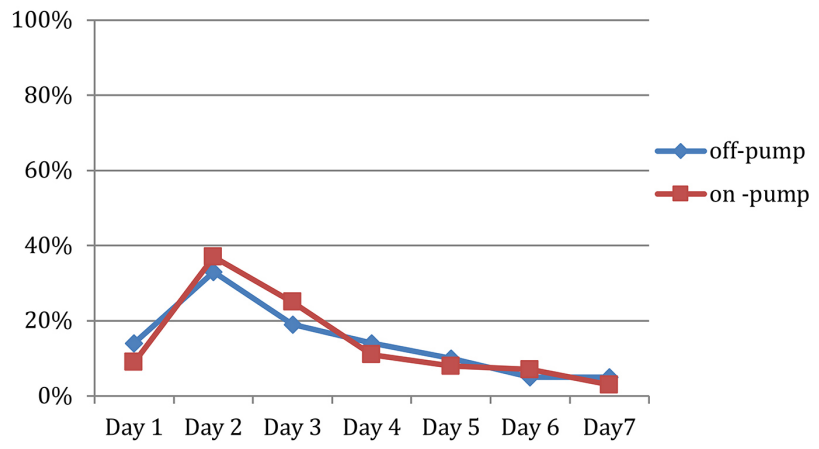

Figure 1. Time to POAF development in on-pump and off-pump CABG surgery patients. POAF, postoperative atrial fibrillation; $C A B G$, coronary artery bypass grafting

$(P=.002)$, positive inotropic support (dopamine) $(P=.001)$, intra-aortic balloon pump $(P=.021)$, chest tube drainage $(P$ $=.001)$, total amount of blood products $(P=.001)$, intubation duration $(P=.001)$, length of ICU stay $(P=.001)$, length of hospital stay $(P=.001)$, and re-exploration $(P=.001)$ (Tables 2 and 3). Furthermore, POAF most commonly developed in the second and third days of surgery and indicated no statistically significant difference between the groups (Figure 1).

The mean time to return to sinus rhythm using medical treatment, which was $19.7 \pm 9.1$ hours, indicating a shorter time for the off-pump group (Figure 2). Seventeen patients were returned to sinus rhythm through electro cardioversion due to sudden hemodynamic instability.

According to the multivariate analysis, the type of operation (OR: $4.85 ; 95 \%$ CI 1.75-6.95; $P=.001)$, number of anastomosis (OR: $3.86 ; 95 \%$ CI 2.61-5.11; $P=.001$ ), right coronary artery or right coronary posterior descending artery graft (OR: 6.85; 95\% CI 4.67-9.03; $P=.001$ ), vasopressor therapy (epinephrine, norepinephrine) (OR: 2.01; 95\% CI 1.09-2.99; $P=.021$ ), operation duration (OR: 4.85; $95 \%$ CI 1.48-3.68; $P=.023$ ), age $>60$ years (OR: $1.22 ; 95 \%$ CI $1.02-1.78 ; P=$ .032 ), hypertension (OR: 2.12; 95\% CI 2.04-6.76; $P=.019$ ), length of hospital stay $>4$ days (OR: 1.31 ; $95 \%$ CI $0.22-2.43$; $P=.041$ ), obstructive sleep apnea syndrome (OR: 1.70; $95 \%$ CI 1.05-2.35; $P=.024)$, were the independent predictors of POAF after CABG (Table 4).

\section{DISCUSSION}

Postoperative atrial fibrillation is one of the most frequent complications within the first week of CABG and is seen in approximately $5 \%$ to $40 \%$ of patients [Andrews 1991]. It still is unclear whether off-pump CABG reduces the rate of POAF. In a meta-analysis including 43 studies, Chen et al reported that off-pump CABG did not significantly reduce POAF, postoperative stroke, and perioperative myocardial infarction [Chen 2012]. However, some authors suggested that the incidence of POAF was lower in patients undergoing off-pump CABG [Hashemzadeh 2013; Sajja 2004; Zoni-Berisso 2014]. 


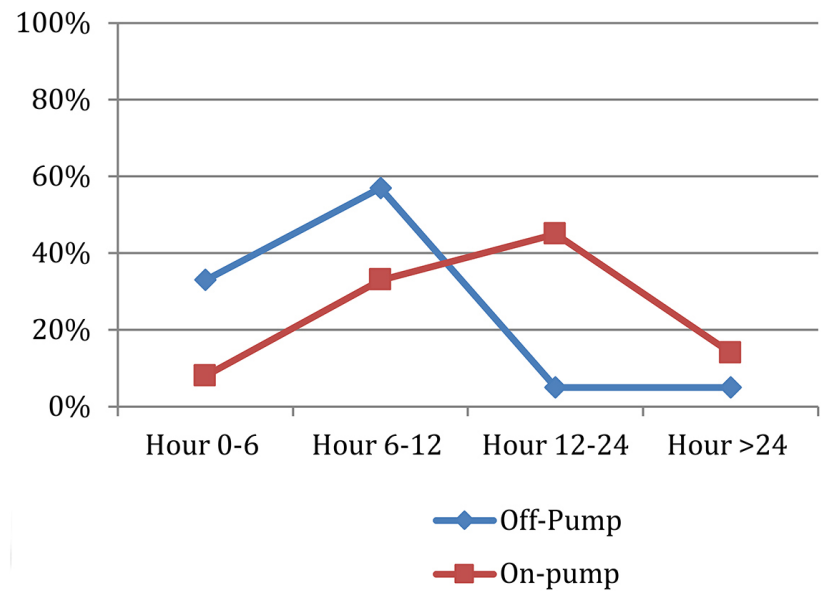

Figure 2. Time to return to sinus rhythm in on-pump and off-pump CABG surgery patients. CABG, coronary artery bypass grafting

We investigated the incidence of POAF in patients undergoing off-pump versus on-pump CABG under $\mathrm{CPB}$ in the present study. We found the incidence of POAF to be $19.86 \%$ and the incidence of POAF to be significantly lower in the off-pump surgery compared with on-pump surgery (10.74\% versus $29.09 \%$, respectively; $P=.001)$. The mean time to POAF development was similar between the groups, although the meantime to return to sinus rhythm was shorter in the off-pump group.

Several independent risk factors were proposed for the development of POAF, following CABG in the literature. Age was suggested to play a key role in the POAF development. The incidence of AF ranged from $0.12 \%$ to $0.19 \%$ in patients aged under 49 years, from $3.7 \%$ to $4.2 \%$ in patients aged $60-70$ years, and from $10 \%$ to $17 \%$ in patients aged $\geq 80$ years [Sajja 2004]. With strong documentation, increasing age was found to be associated with degenerative alterations of the atrial myocardium, reduced response to oxidative stress, and altered electrical properties of the sinoatrial node and atrioventricular node [Allessie 2001]. We also found a significant increase in the rate of POAF in patients aged $\geq 60$ years $(P=0.032)$ in the present study. Although being male is a well-defined risk factor for POAF development [Lee 2017], and the present study also finds a significant correlation between sex and POAF development $(P=.041)$.

Hypertension is a major preventable cardiovascular risk factor for POAF development [Lau 2012]. In line with the literature, we found a significant effect of hypertension on POAF development $(P=.001)$. This finding indicates that blood pressure control is an effective way of POAF prevention as hypertension is an independent risk factor for POAF. Furthermore, several studies support a role for sympathetic activation in the pathogenesis of AF following cardiac surgery [Dzeshka 2017; Chelazzi 2011; Workman 2010]. Similarly, the present study also showed that vasopressor therapy (i.e., epinephrine, norepinephrine) significantly increased the incidence of $\operatorname{POAF}(P=.002)$.

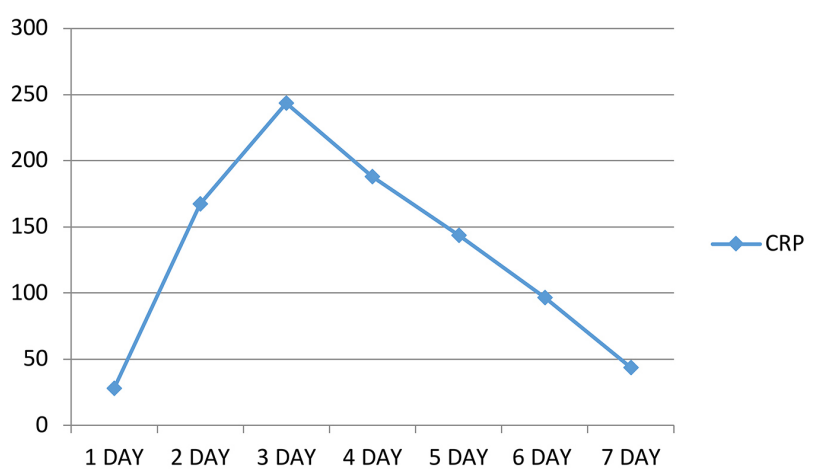

Figure 3. CRP levels $(\mathrm{mg} / \mathrm{dl})$ in on-pump and off-pump CABG surgery patients. CRP, C-reactive protein

Additionally, recent studies demonstrated patients with OSAS have a higher incidence of POAF compared with controls, and preoperative continuous positive airway pressure decreased the incidence of POAF in this patient population [Patel 2010; Mooe 1996]. Likewise, we also found OSAS to be an independent risk factor that increased the incidence of $\operatorname{POAF}(P=.024)$.

There also are several studies investigating the preventive effect of medications for POAF in patients undergoing CABG [Wang 2017]. Among them, statins are the most commonly used drugs in the treatment of atherosclerotic heart disease. Statins exert their effect mainly by lowering serum cholesterol levels. However, additional favorable effects of statins on the cardiovascular system were recently found as well as their effects on cholesterol synthesis [Diamantis 2017]. Statins also were found to decrease inflammatory markers, irrespective of their lipid-lowering effect [Diamantis 2017; Lefer 2002]. In a meta-analysis including 17 studies, the relationship between preoperative statin use and incidence of AF, early cardiac complications such as POAF, myocardial infarction, and myocardial injury, and inflammatory markers (i.e., cytokines, C-reactive protein $[\mathrm{CRP}]$ ) in patients undergoing $\mathrm{CABG}$ and preoperative statin use was associated with a significant decrease in the POAF incidence [Diamantis 2017; Lefer 2002; An 2017].

Recent studies also demonstrated ACE inhibitors, which currently are widely used to decrease mortality in patients with heart failure and myocardial infarction [Berliner 2018]. Mortality partly is related to arrhythmias in selected patients, so it is thought that ACE inhibitors may have antiarrhythmic effects. There also are clinical studies suggesting that angiotensin receptor blockers (ARBs) or ACE inhibitors decrease the incidence of $\mathrm{AF}$ in patients with chronic heart failure and myocardial infarction [Pedersen 1999]. In a meta-analysis, preoperative beta-blocker use was associated with reduced incidence of AF following CABG, therefore, it should not be discontinued [Burgess 2006]. However, the authors highlighted preoperative beta-blocker for the prevention of POAF to be insufficient when used alone [Burgess 2006]. Statin and ACE inhibitor usage doses and molecules were different and statistical analysis could not be made since this study was retrospective. 
Table 4. Risk Factors for POAF development

\begin{tabular}{|c|c|c|c|c|}
\hline Number of anastomosis $\geq 3$ & .001 & 3.86 & 2.61 & 5.11 \\
\hline Right coronary artery or right coronary posterior descending artery graft & .001 & 6.85 & 4.67 & 9.03 \\
\hline Operation duration $(\mathrm{min})>100 \mathrm{~min}$ & .023 & 2.58 & 1.48 & 3.68 \\
\hline Age $>60$ & .032 & 1.22 & 1.02 & 1.78 \\
\hline Hypertension & .019 & 2.12 & 2.04 & 6.76 \\
\hline Length of hospital stay (day) $>4$ day & .041 & 1.31 & 0.22 & 2.43 \\
\hline Obstructive sleep apnea syndrome & .024 & 1.70 & 1.05 & 2.35 \\
\hline
\end{tabular}

Model $\chi^{2}: 11,25, P<.05$; success rate $=86.90 \%$; Nagelkerke $\mathrm{R}^{2}=0.652$

Data are given in mean \pm SD or in number. NS, not significant; COPD, chronic obstructive pulmonary disease; LVEF, left ventricular ejection fraction; CRP,

C-reactive protein; CPB, cardiopulmonary bypass; ICU, intensive care unit; $\mathrm{ACE}$, angiotensin-converting enzyme; OR, odds ratio; Cl, confidence interval; POAF, postoperative atrial fibrillation

C-reactive protein is an acute inflammatory protein secreted by the liver [Fakanya 2014]. Previous studies found CRP to be associated with increased cardiovascular complications [Fakanya 2014; Yehc 2001; Liuzzo 1994]. In their study titled Atorvastatin for Reduction of Myocardial Dysrhythmia after Cardiac Surgery (ARMYDA-3), Patti et al reported patients with POAF to have the highest CRP levels following surgery [Patti 2006]. In the aforementioned study, multivariate analysis revealed increased CRP levels above median values to be significantly correlated with POAF development. In another study investigating the effect of CRP on POAF development, postoperative CRP levels and postoperative arrhythmias simultaneously reached their peak (i.e., on postoperative days 2 and 3) [Bruins 1997]. Similarly, the highest CRP levels and POAF most commonly were observed on the second, third, and fourth postoperative days in our study. However, we found no significant correlation between the elevated CRP levels and POAF development or sinus rhythm (Figure 3).

There is no dramatic decline in the POAF incidence, and POAF still is a major concern of morbidity and mortality for patients undergoing CABG despite the developments in the surgical techniques and therapeutic modalities. POAF previously was associated with hemodynamic instability, increased cerebrovascular disease, increased treatment expenditures, and prolonged hospital stays [Kosuma 2018]. None of the patients had any thromboembolic complications in our study. There also was no significant difference in the in-hospital mortality among the patients with POAF and those with sinus rhythm. However, the patients with POAF had a significantly prolonged hospital stay in our study $(P=.04)$.

In line with the literature, our study results indicate that $\mathrm{CPB}$ is an important risk factor for POAF. Nonetheless, there still is a need for further studies investigating the etiology of POAF considering CPB, hypothermia and cardioplegic arrest-related factors, and other risk factors including reperfusion injury, cardiac manipulation, postoperative pericardial effusion, electrolyte imbalance, intra- and postoperative catecholamine discharge, and histopathological atrial alterations with advanced age, which all are common characteristics of on-pump and off-pump surgery. Moreover, further studies for predicting and preventing POAF to reduce morbidity and mortality are required in this patient population.

There were some limitations to this study. The elimination of CPB-related adverse effects during off-pump surgery, a lower number of distal anastomoses and right coronary artery or right coronary posterior descending artery grafts in patients undergoing off-pump CABG, less diffuse coronary artery disease, a lower amount of chest tube drainage and blood products, and lower use of vasopressor therapy, positive inotropic support, and IABP might have affected the results.

As a result, off-pump CABG yielded a lower number of POAF compared with on-pump surgery. Although all POAF patients, irrespective of the type of surgery, were returned to sinus rhythm with pharmacological or non-pharmacological treatment; the meantime to return to sinus rhythm was relatively shorter in the patients undergoing off-pump CABG. Based on multivariate analysis and these findings, we conclude that the CABG on-pump may be associated with the development of POAF, and we recommend considering off-pump CABG in select situations to minimize the risk of POAF.

\section{REFERENCES}

Allessie MA, Boyden PA, Camm AJ, et al. 2001. Pathophysiology and prevention of atrial fibrillation. Circulation. 103:769-77.

An J, Shi F, Liu S, Ma J, Ma Q. 2017. Preoperative statins as modifiers 
of cardiac and inflammatory outcomes following coronary artery bypass graft surgery: a meta-analysis. Interact Cardiovasc Thorac Surg. 25(6):958-965.

Andrews TC, Reimold SC, Berlin JA, Antman EM. 1991. Prevention of supraventricular arrhythmias after coronary artery bypass surgery. A meta-analysis of randomized control trials. Circulation. 84(5 Suppl):III236-III244.

Berliner D, Hallbaum M, Bauersachs J. 2018. Current drug therapy for heart failure with reduced ejection fraction. Medikamentöse Therapie der Herzinsuffizienz mit reduzierter systolischer LV-Funktion. Herz. 43(5):383-391.

Bruins P, te Velthuis H, Yazdanbakhsh AP, et al. 1997. Activation of the complement system during and after cardiopulmonary bypass surgery: postsurgery activation involves C-reactive protein and is associated with postoperative arrhythmia. Circulation. 96(10):3542-3548.

Burgess DC, Kilborn MJ, Keech AC. 2006. Interventions for prevention of post-operative atrial fibrillation and its complications after cardiac surgery: a meta-analysis. Eur Heart J. 27(23):2846-2857.

Chelazzi C, Villa G, De Gaudio AR. 2011. Postoperative atrial fibrillation. ISRN Cardiol 2011:203179.

Chen YB, Shu J, Yang WT, et al. 2012. Meta-analysis of randomized trials comparing the effectiveness of on-pump and off-pump coronary artery bypass. Chin Med J (Engl). 125(2):338-344.

Diamantis E, Kyriakos G, Quiles-Sanchez LV, Farmaki P, Troupis T. 2017. The Anti-Inflammatory Effects of Statins on Coronary Artery Disease: An Updated Review of the Literature. Curr Cardiol Rev. 13(3):209-216.

Dzeshka MS, Shahid F, Shantsila A, Lip GYH. 2017. Hypertension and Atrial Fibrillation: An Intimate Association of Epidemiology, Pathophysiology, and Outcomes. Am J Hypertens. 30(8):733-755.

Fakanya WM, Tothill IE. 2014. Detection of the inflammation biomarker C-reactive protein in serum samples: towards an optimal biosensor formula. Biosensors (Basel). 4(4):340-357.

Gasparova I, Kubatka P, Opatrilova R, et al. 2017. Perspectives and challenges of antioxidant therapy for atrial fibrillation. Naunyn Schmiedebergs Arch Pharmacol. 390(1):1-14.

Hashemzadeh K, Dehdilani M, Dehdilani M. 2013. Does Off-pump Coronary Artery Bypass Reduce the Prevalence ofAtrial Fibrillation? J Cardiovasc Thorac Res. 5(2):45-49.

Hogue CW Jr, Hyder ML. 2000. Atrial fibrillation after cardiac operation: risks, mechanisms, and treatment. Ann Thorac Surg. 69(1):300-306.

Kosuma P, Wachirasrisirikul S, Jedsadayanmata A. 2018. Attributable Costs of Postoperative Atrial Fibrillation among Patients Undergoing Cardiac Surgery. Cardiol Res Pract. 2018:3759238.

Lau YF, Yiu KH, Siu CW, Tse HF. 2012. Hypertension and atrial fibrillation: epidemiology, pathophysiology and therapeutic implications. J Hum Hypertens. Oct;26(10):563-9.
Lee SH, Lee H, Park JK, et al. 2017. Gender Difference in the LongTerm Clinical Implications of New-Onset Atrial Fibrillation after Coronary Artery Bypass Grafting. Yonsei Med J. 58(6): 1119-1127.

Lefer DJ. 2002. Statins as potent anti-inflammatory drugs. Circulation. 106: $2041-2042$.

Liuzzo G, Biasucci LM, Gallimore JR, Grillo RL, Rebuzzi AG, Pepys MB, Maseri A. 1994. The prognostic value of $\mathrm{C}$ - reactive protein and serum amyloid a protein in severe unstable angina. N Engl Med. $331: 417-24$.

Maesen B, Nijs J, Maessen J, Allessie M, Schotten U. 2012. Postoperative atrial fibrillation: a maze of mechanisms. Europace. 14(2):159-174.

Mooe T, Gullsby S, Rabben T, Eriksson P. 1996. Sleep-disordered breathing: a novel predictor of atrial fibrillation after coronary artery bypass surgery. Coronary Artery Disease. 7(6):475-478.

Patel D, Mohanty P, Di Biase L, et al. 2010. Safety and efficacy of pulmonary vein antral isolation in patients with obstructive sleep apnea: the impact of continuous positive airway pressure. Circulation: Arrhythmia and Electrophysiology. 3(5):445-451.

Patti G, Chello M, Candura D. 2006. Randomized Trial of Atorvastatin for Reduction of Postoperative Atrial Fibrillation in Patients Undergoing Cardiac Surgery Results of the ARMYDA-3 (Atorvastatin for Reduction of Myocardial Dysrhythmia After cardiac surgery) Study. Circulation. 114:1455-1461.

Pedersen OD, Bagger H, Kober L, Torp- Pedersen C. 1999. Trandolapril reduces the incidence of atrial fibrillation after acute myocardial infarction in patients with left ventricular disfunction. Circulation. 100: 376 -380 .

Sabzi F, Zokaei AH, Moloudi AR. 2011. Predictors of atrial fibrillation following coronary artery bypass grafting. Clin Med Insights Cardiol. 5.67-75.

Sajja LR, Mannam G, Sompalli S, et al. 2004. Does multi- vessel off-pump coronary artery bypass graftingreduce postoperative morbidity compared to on-pump CABG? Ind J Thorac Cardiovasc Surg. 20:173-177.

Wang X, Yao L, Ge L, et al. 2017. Pharmacological interventions for preventing post-operative atrial fibrillation in patients undergoing cardiac surgery: a network meta-analysis protocol. BMJ Open. 7(12):e018544.

Workman AJ. 2010. Cardiac adrenergic control and atrial fibrillation. Naunyn Schmiedebergs Arch Pharmacol. 381:235-49.

Yanagawa B, Puskas JD. 2016. Off-Pump Coronary Artery Bypass Grafting Operative Techniques in Thoracic and Cardiovascular Surgery Volume 21, Issue 1, spring Pages 2-19.

Yehc ET, Anderson HV, Pasceri V, Willerson JT. 2001. C-Reactive Protein Linking Inflammation to Cardiovascular Complications. Circulation. 104; 974-975.

Zoni-Berisso M, Lercari F, Carazza T, Domenicucci S. 2014. Epidemiology of atrial fibrillation: European perspective. Clin Epidemiol. $6.213-220$. 